\title{
Secondary Electron Imaging - Doing it Better
}

\author{
D.C. Joy, ${ }^{* * * *}$ and B.J. Griffin, ${ }^{* *},{ }^{* * *}$
}

* Dept. of Materials Science and Engineering, University of Tennessee, Knoxville, TN37916

** Centre for Microscopy, Characterization and Analysis (M010), University of Western Australia, Crawley, WA 6009, Australia

*** Center for NanoPhase Materials Science, Oak Ridge National Laboratory, Oak Ridge, TN 37831

Secondary electron (SE) imaging is the most widely employed mode for operating the SEM. This is because the SE, defined as being those electrons with energies between 0 and $50 \mathrm{eV}$, are capable of spatial resolution limited only by the performance of the SEM itself, and produce readily interpretable topographic contrast over a range of size scales from centimeters to nanometers. A closer inspection, however, reveals the complexity of this imaging mode and the ambiguities and uncertainties inherent in its interpretation.

SE are produced by the interaction of the incident electron beam with the sample and emerge from a region a few nanometers in extent beneath the irradiated surface. The SE signal is not however a single entity because invariably it contains several distinct components each of which carries different information. The SE1 signal, which is typically 10 to $20 \%$ of the total signal, carries surface information with a resolution determined by the spot size and sample material but potentially in the nanometer range; SE2 signal (20 to $40 \%$ of the total) are produced by exiting backscattered electrons and so is dominated by information from features extending down to about $1 / 3^{\text {rd }}$ of the beam range in the sample and have a resolution of the order of the escape range; and SE3 signal, typically $50 \%$ of the total SE yield, which are generated by scatter in and around the sample chamber and so are unrelated to the sample and have a resolution of the order of the specimen chamber dimensions. Taken together these SE components provide an image with valuable detail and high contrast, but randomly assembled from three quite different types of signal information. The SE3 signal is random noise and can, and should, be eliminated by enlargement of the sample chamber and by the removal of all hardware items except the detectors and stage. The fact that such optimization is rarely attempted or applied leads to a significant drop in the signal to noise ratio (SNR) achieved and unnecessarily degrades the SEM imaging performance.

Both the SE1 and SE2 signals contain meaningful, but different, image information. It is usually assumed that the SE1 and SE2 components cannot be separated because they co-exist within the same energy range $0-50 \mathrm{eV}$ but this is not correct. Comparisons of conventional SE spectra with ion induced (iSE) spectra, for which the backscattering contribution is negligible, show that the iSE and eSE spectra are essentially identical for energies below about $15 \mathrm{eV}$. Above $15 \mathrm{eV}$ the iSE spectrum decays rapidly while the eSE only falls slowly towards a minim value at about $50 \mathrm{eV}$. As shown in figure (1) this makes it possible to positively identify the SE1 and SE2 components of the signal

Separating the SE1 from the SE2 component could afford several enhancements in performance. First, a significant improvement in SE1 SNR is possible if the SE2 (and SE3) components are eliminated. Based on the estimated intensities of the other contributions relative to SE1 it is estimated that the SE1 SNR could be enhanced by a factor of nearly $2 x$ for the same incident beam 

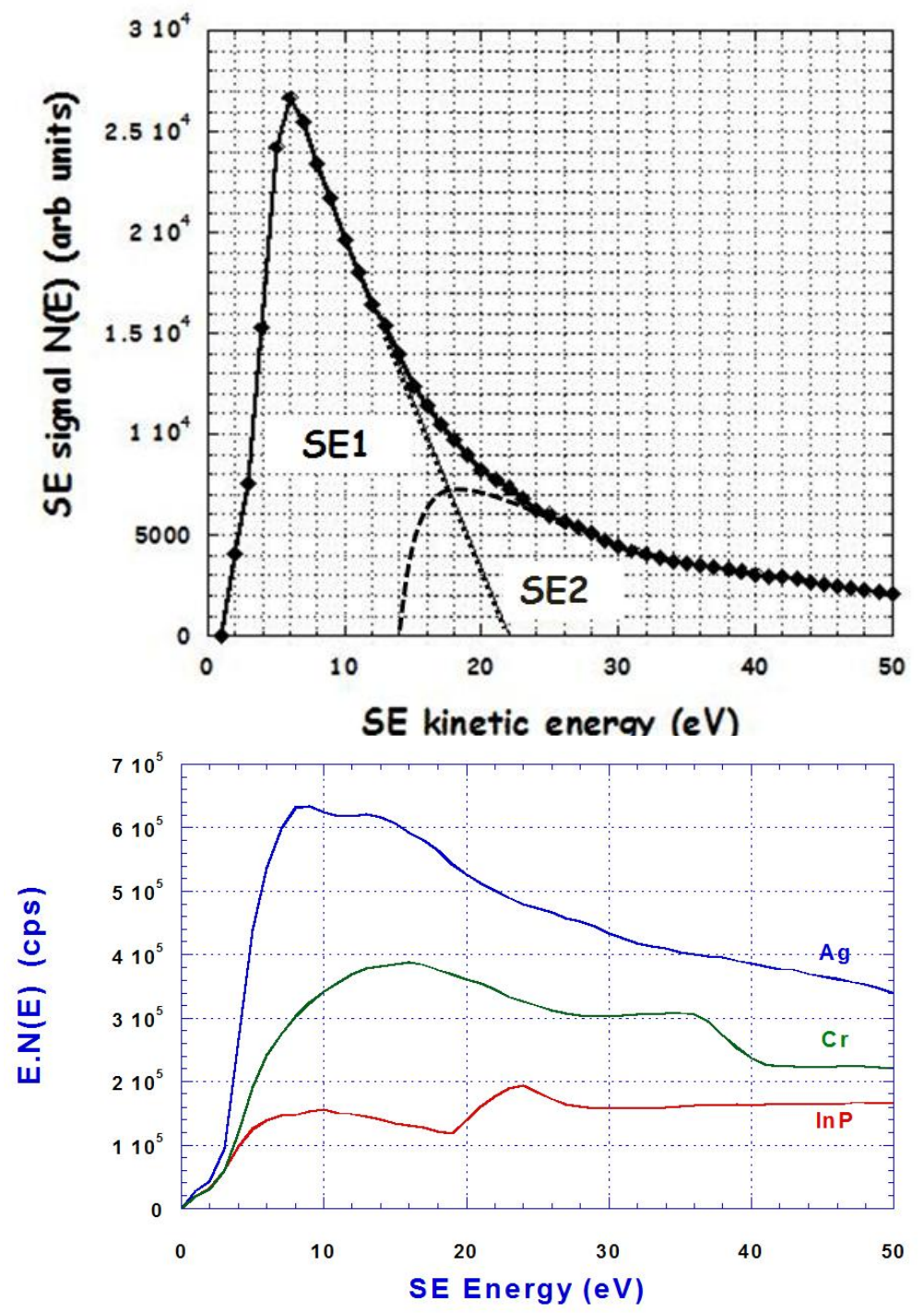

FIG 1. SE1 and SE2 components of the eSE spectrum of silicon deduced by comparison with the corresponding ion-induced (iSE) spectra
FIG 2. Experimental SE spectra from $\mathrm{Ag}, \mathrm{Cr}$, and InP . The E.N(E) vs E plot reveals the differences between the spectra from elements, and from compounds

current by setting the analyzer to optimize the energy window for SE1 contrast while eliminating the SE2 component and suppressing the SE3 by enhanced chamber design.

Once separated from the SE2 by filtering the SE1 mode would provide probe size limited spatial resolution with no degradation or loss of contrast due to BSE components. Adjustments of the SE1 energy acceptance window within the spectrometer could also further optimize imaging by selecting the region around the peak energy for SE1 emission (typically 5-6eV) and by eliminating surface contamination and charging effects. Finally, because SE1 spectra (figure 2) are unique to the element, or compound, from which they come, even if the specimen surface is contaminated, and have a yield five to six orders of magnitude higher than for X-rays, a new and highly efficient form of microanalysis would become available.

\section{References}

[1] We are grateful to Dr. H Meyer (ORNL) for experimental SE and ion induced iSE spectra 